\title{
A provable secure key-escrow-free identity-based signature scheme without using secure channel at the phase of private key issuance
}

\author{
SUBHAS CHANDRA SAHANA ${ }^{1, *}$, MANIK LAL DAS ${ }^{2}$ and BUBU BHUYAN ${ }^{1}$ \\ ${ }^{1}$ Department of Information Technology, North Eastern Hill University, Shillong 793022, India \\ ${ }^{2}$ Faculty Block 2, Dhirubhai Ambani Institute of Information and Communication Technology (DA-IICT), \\ Gandhinagar 382007, India \\ e-mail: subhas.sahana@gmail.com; maniklal@gmail.com; b.bhuyan@gmail.com
}

MS received 5 November 2017; revised 28 November 2018; accepted 27 February 2019; published online 6 May 2019

\begin{abstract}
The identity-based cryptosystems furnish us with simplest key management procedures. Yet, they have a very slow adoption in cryptography due to the key escrow problem and the necessity of a secure channel between the user and the Private Key Generator (PKG) to transmit the created private key to the user. In this paper, we propose an identity-based signature scheme that not only solves the key escrow problem but also eliminates the requirement of the secure channel. The proposed scheme is secure against existential forgery under adaptively chosen message and ID attacks in the random oracle model assuming the hardness of the Computational Diffie-Hellmann Problem (CDHP). Furthermore, we compare the efficiency of our scheme to that of a similar established scheme.
\end{abstract}

Keywords. Digital signature; identity-based cryptosystem; key escrow problem; computation Diffie-Hellman problem (CDHP); adaptively chosen message and identity attack.

\section{Introduction}

Different cryptosystems have been introduced with an aim of providing security to electronic data in this digital world. Each user in a traditional Public Key Cryptosystem (PKC) [1,2] must have a certificate, binded with his/her public key along with the signature of the Certificate Authority (CA) before getting involved in any event (for example, message encrypting/signature verification process). The CA's signature is utilized on the certificate in order to preserve the authenticity of the public key of a corresponding user. A user's identity along with his/her public key must be recorded by the CA so that it can be used later for validation of the user's public key. Moreover, apart from signing and storing these certificates, all other certificate management activities must be performed by the CA, such as certificate issuance, certificate renewal, certificate revocation, etc. To encrypt or verify a digital message, one needs a verified public key, embedded in a certificate that must have gone through a process called certificate verification. Actually, the overhead of this process depends on the infrastructure of the network, which proves that it is inefficient in terms of storage and involved computational cost.

In 1984, identity-based cryptosystem (IBC) was introduced by Shamir [3] with an aim of overcoming the

*For correspondence difficulties faced in the traditional PKC. The key management process in IBC is very simple compared with the traditional PKC. The public key can be derived easily from a unique identity of the user. Hence, no certificates are required, which results in removing the certificate verification process and certificate management process and makes it efficient in terms of involved computational cost as well as storage cost. A trusted third party, known as the Private Key Generator (PKG), generates the private key for the user.

After the pioneering work proposed by Shamir [3], many identity-based signature schemes [4-6] have been proposed. Here, it is to be noted that finding a practical identity-based encryption/signature scheme remained an open problem until 2001. In 2001, Boneh and Franklin proposed the first identitybased encryption scheme [7] based on Weil Pairing applied over elliptic curve. Afterwards, many identity-based signature schemes [8] have been proposed based on bilinear pairing.

All the identity-based signature schemes in [8-11] suffer from an inherent problem, called key escrow problem as the PKG knows the user's private key which is computed by the PKG only and can forge a signature of a message for a user. Moreover, all those identity-based signature schemes require a secure channel between the PKG and the user to transmit the private key.

To overcome these two problems of the IBC, many approaches were proposed and intensively investigated. A 
solution was proposed for eliminating the key escrow problem by Boneh and Franklin [7], where the user's private key is computed in a threshold manner by multiple trusted authorities. However, it is a fact that multiple identity verifications of a user by multiple authorities are computationally quite inefficient. In [12], an approach was proposed where a new private key is generated by adding multiple private keys but this was not a successful approach as the PKGs have no countermeasure against illegal usage of user's private keys. Gentry [13] proposed a scheme that is based on certificates to eliminate the secure channel requirement as well as the key escrow using some user-chosen secret information. In 2003, Al-Riyami and Paterson [2] introduced the concept of certificateless cryptosystem. Though an identity-based signature scheme fitted in certificateless cryptosystem eliminates the key escrow problem, the requirement of a secure channel between users and a trusted authority such as PKG to transmit partial-private key is still there. Lee et al [14] proposed a scheme to eliminate the key escrow problem; however, it is not so efficient due to its involved computational cost as the issued private key's privacy is protected by multiple key privacy authorities.

\section{Our contribution}

In this paper, we propose a key-escrow-free identity-based signature scheme without using a secure channel at the phase of private key issuance. We use the binding-blinding technique [15] to overcome the key escrow as well as to eliminate the requirement of a secure channel at the phase of private issuance. The presented scheme is based on bilinear pairing and fitted in the model proposed by Das [16].

\section{Organization of the paper}

The paper is organized as follows. Section 2 provides some preliminaries that are required to be understood beforehand. We propose a security model for the signature model, proposed by Das [16] in section 3. In section 4, we propose an identity-based signature scheme fitted in the model, based on binding-blinding technique [16]. Security analysis of the proposed scheme in the Random Oracle Model (ROM) is done in section 5, In section 6, efficiency analysis is discussed. Finally, we conclude our work in section 7 .

\section{Preliminaries}

In this section, we present some preliminaries including basic mathematical background and computational problems that are required for our work.

\subsection{Group}

A group $G$ is a set together with an operation " $*$ " that combines any two elements $a$ and $b$ to form another element, denoted by $a * b$. The group $(G, *)$ must satisfy the following conditions.
1. Closure: For all $a, b \in G$, the value of $a * b \in G$.

2. Associative: For all $a, b$ and $c \in G,(a * b) * c=a *(b * c)$.

3. Identity element: An element $e$ exists in $G$ such that every element $b \in G$ satisfies the condition $e * b=b *$ $e=b$. The element $e$ is called the identity element.

4. Inverse element: For every single element $b \in G$, there exists an element $c$ such that $b * c=e$, i.e. identity element.

Abelian group: A group is called abelian group if it follows the extra property called commutative property (i.e., $a * b=b * a$ for all $a, b \in G)$. All cyclic groups are abelian, but an abelian group is not necessarily cyclic.

Multiplicative group: A group is called multiplicative group if the group operation is multiplication, denoted by $*$. The identity element of a multiplicative group is 1 and the inverse of the element $a$ is denoted by $a^{-1}$.

Additive group: A group is called additive where the group operation is addition, denoted by + . The identity element and inverse of an element $a$ of the additive group are identified by 0 and $-a$, respectively.

\subsection{Field}

A set with two operations " + " and " $*$ " is called a field $\mathbb{F}$ if the following condition holds for all $a, b, c \in F$

1. Closure: $a+b$ and $a * b$ must $\in F$.

2. Associative: $a+(b+c)=(a+b)+c$ and $a *(b * c)=$ $(a * b) * c$.

3. Commutative: $a+b=b+a$ and $a * b=b * a$.

4. Additive and multiplicative identity: There exists an additive identity denoted by " 0 " such that $0+a=$ $a+0=a$ and is known as the additive identity. In addition to this, there exists another element, denoted by " 1 ", such that $1 * a=a * 1=a$ and is known as the multiplicative identity.

5. Additive and multiplicative inverse: An inverse element $b$ exists for an element $a$ such that $a+b=0$ (identity element) in case of addition and $a * b=1$ (identity element) in case of multiplication. Here, $b$ is called additive inverse of $a$ in case of addition operation and multiplicative inverse of $a$ in case of multiplication operation.

6. Distributive: Multiplication must be distributive over addition, i.e. $\mathrm{a} *(b+c)=(a * b)+(a * c)$.

\subsection{Elliptic curve}

An elliptic curve [17] over a field $F$ is a non-singular cubic curve with the property that the curve does not have repeated roots. The general equation of an elliptic curve is given as follows: 


$$
y^{2}+b_{1} x y+b_{2} y=x^{3}+b_{3} x^{2}+b_{4} x+b_{5}
$$

where the coefficients $b_{i} \in F$ for $i=1,2,3, \ldots, 5$. The elliptic curve $E$ over the finite field $F$, denoted by $E(F)$, consists of all the points $P(x, y)$ on the curve such that $x, y \in$ $F$ along with a special point, called a "Point-at-Infinity", denoted by $\mathcal{O}$. Moreover, the set of points $P \in E(F)$ along with $\mathcal{O}$ point forms an abelian additive group where $\mathcal{O}$ is the additive identity. Let $F_{q}$ denote the finite field of order $q$ with the characteristic $p$. Without loss of generality, the elliptic curve equation over $F_{q}[17,18]$ can be simplified to the form

$$
y^{2}=x^{3}+a x+b
$$

1. Finding additive inverses: For each point $P(x, y) \neq \mathcal{O}$ and $P \in E(F)$, the inverse element is $-P(x,-y)$.

2. Adding two points: The rules for adding two points $P, Q \in E\left(F_{p}\right)$ over the Galois prime field, i.e. $G F(p)$, are given as follows:

- If $P=\left(x_{1}, y_{1}\right), \quad Q=\left(x_{2}, y_{2}\right), \quad Q \neq P, \quad$ then $R\left(x_{3}, y_{3}\right)=P+Q$ is calculated as

$x_{3}=\left(\frac{y_{2}-y_{1}}{x_{2}-x_{1}}\right)^{2}-x_{1}-x_{2}$, and

$y_{3}=\left(\frac{y_{2}-y_{1}}{x_{2}-x_{1}}\right)\left(x_{1}-x_{3}\right)-y_{1}$.

- If $Q=P$, then $R\left(x_{3}, y_{3}\right)=P+P=2 P$ can be calculated as

$$
\begin{aligned}
& x_{3}=\left(\frac{3 x_{1}^{2}+a}{2 y_{1}}\right)^{2}-2 x_{1}, \text { and } \\
& y_{3}=\left(\frac{3 x_{1}^{2}+a}{2 y_{1}}\right)\left(x_{1}-x_{3}\right)-y_{1}
\end{aligned}
$$

where $a$ is the coefficient of $x$ in Eq. (2).

- Multiplying a point by a constant: Multiplying a point by a constant $n$ is defined as $n$-fold addition of $P$, i.e., $n P=P+P+P+\cdots+P$ (n-times) [17].

\subsection{Bilinear map and complexity assumption}

Let $G_{1}$ be an additive group of prime order $q$ and $G_{2}$ be a multiplicative group of the same order. Let $P$ denote a generator of $G_{1}$ and $e$ be a computable map $e: G_{1} \times G_{1} \rightarrow$ $G_{2}$ with the following properties.

1. Bilinear: $e(a P, b Q)=e(P, Q)^{a b}$ for all $P, Q \in G_{1}$, $a, b \in Z_{q}$.

2. Non-degenerate: $e(P, P) \neq 1 \in G_{2}$.

3. There is an algorithm to compute the map $e$ efficiently.

- Discrete Logarithm Problem (DLP): Two prime numbers $p$ and $q$ are taken such that $q$ divides $(p-1)$. Let $g \in_{R} Z_{q}^{*}, g$ is of order $q$ and $y$ is a random element generated by $g$. If $g^{x}=y \bmod p$, the probability of finding $x$ using any polynomial time algorithm is negligible.

- Elliptic Curve Discrete Logarithm Problem (ECDLP): For a given elliptic curve $\mathrm{E}\left(F_{q}\right)$, the points $P$ and $Q$ such that $P \in E\left(F_{q}\right)$ and $Q=x . P$, the probability of finding $x$ using any polynomial time algorithm is negligible.

- Computational Diffie-Hellman problem (CDHP): The CDHP is to compute $a b P$ for given $(P, a . P, b . P)$. For all $a, b \in \mathbb{Z}_{q}^{*}$ the advantage of solving CDHP in $G_{1}$ using any probabilistic polynomial time algorithm, defined as $A d v_{P, G_{1}}^{C D H P}=\operatorname{Pr}[\beta(P, a . P, b . P, a . b . P)=1]$, is negligible.

\section{- Decisional Diffie-Hellman Problem (DDHP)}

For $a, b, c \in_{R} \mathbb{Z}_{q}^{*}$, if $P, a P, b P, c P$ is given, to decide whether $c \equiv a b \bmod q$ is known as Decisional DiffieHellman Problem. The DDHP is not a hard problem as bilinear pairing can be used to solve this decision problem in polynomial time.

- Gap Diffie-Hellman (GDH) group: A group $G$ is called a Gap Diffie-Hellman (GDH) group if DDHP can be solved in polynomial time but no probabilistic algorithm can solve CDHP with non-negligible advantage within polynomial time in $G$.

\subsection{Map-to-point hash function}

It is a special hash function using which a message is converted to a point with particular order $p$ on an elliptic curve. The message is first converted into an integer and then mapped to an elliptic curve point.

Let $\mathrm{E}$ be an elliptic curve. It is defined using the equation $y^{2}=f(x)$ and has order $l$. The hash function $h:\{0,1\}^{*} \rightarrow$ $F_{q} \times\{0,1\}$ is a one-way collision-resistant hash function used in map-to-point hash function. The basic map-to-point hash function [7] is shown in algorithm 1.

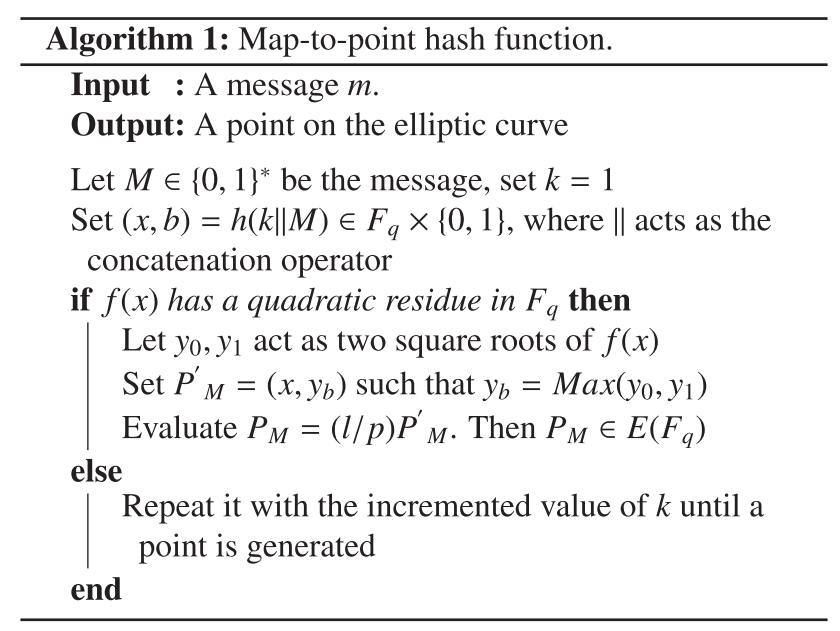




\subsection{Binding-blinding technique [15]}

In our proposed scheme, this technique is adopted to eliminate the key escrow problem and the necessity of a secure channel. In 2010, this technique was embedded with the Hess's identity-based signature scheme [19] to enhance the scheme. In 2015, the author also proposed an identitybased multi-signature scheme [20] from the enhanced scheme of Hess's scheme [19]. As per the technique, a user submits a partial-private key request to the PKG by sending four binding parameters along with user identity over an insecure channel. All four binding parameters hide the two blinding factors, which are kept secret with the user. PKG then validates the received parameters along with the user identity. Upon successful validation of the binding parameters, PKG creates the partial-private key using its own secret key called master secret key and sends back the generated partial-private key to the user over an insecure channel. This step adopts some mechanism such as e-mail confirmation to prevent an unregistered ID attack in the scheme. Upon receiving the partial-private key the user validates sent partial-private key data and calculates the private key. Finally, we note that the binding-blinding technique is used to generate user's private key from the user's public key along with two secret components, one secret component from the user's side and another secret component from the PKG side. The user's PKG process is shown in figure 1 .

\section{Security model}

The standard notion of security for a signature scheme is called existential unforgeability against adaptive chosen message attack defined by Pointcheval and Jacques [21]. In 2010, a VIDS model was proposed by Das [16]; however, the author did not present a formal security model or define any adversary type for his proposed model. In this section, a formal security model in the ROM is proposed.

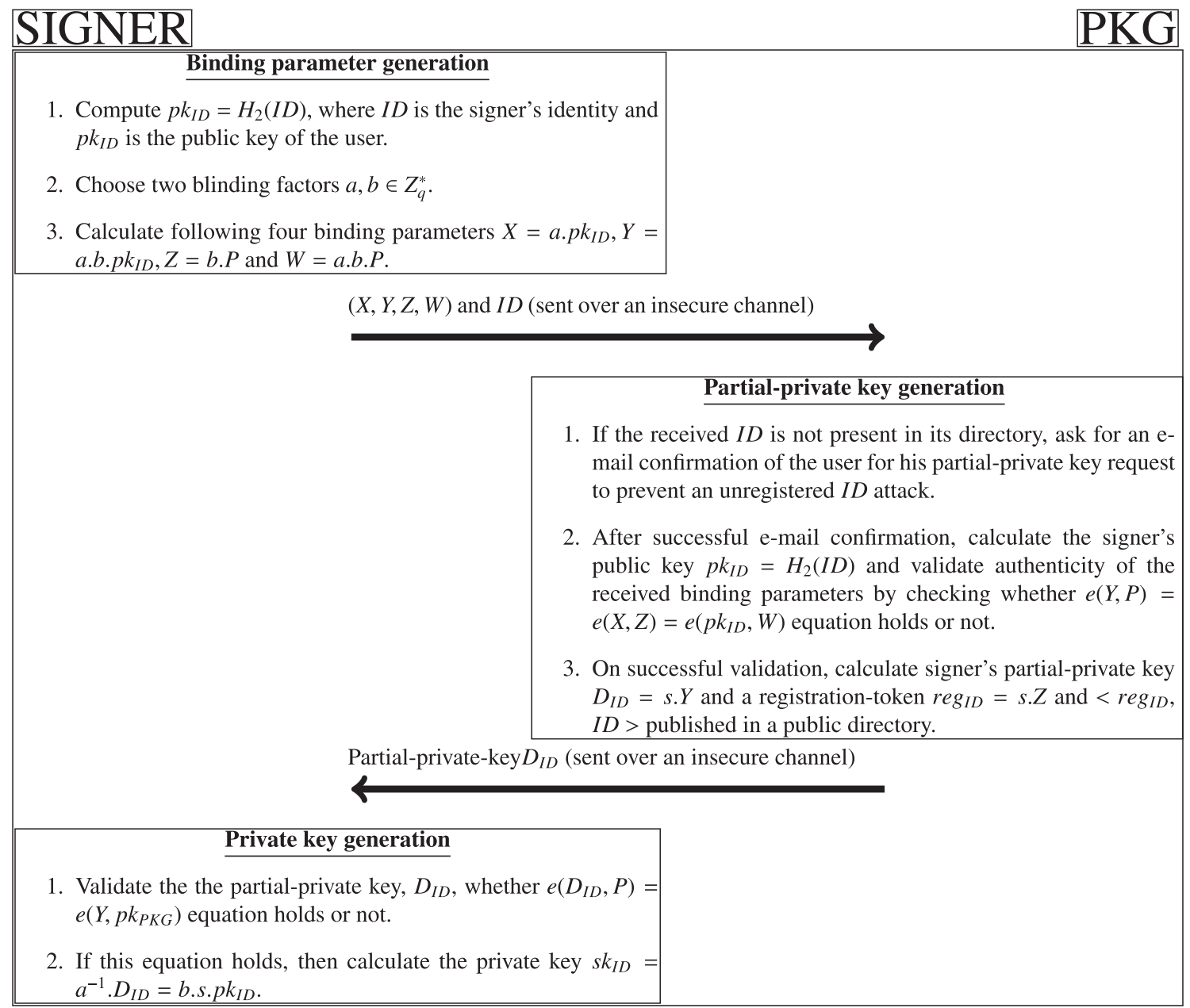

Figure 1. Private key generation for the user. 


\subsection{Adversaries}

We can define two types of adversaries (Type I and Type II) for the VIDS scheme just like those in a CertificateLess Public Key Cryptosystem (CLPKC) [22]. Type I adversary is a dishonest user who can replace the public key of the original signer but does not have the access to the master secret key, and Type II adversary is the malicious PKG who is armed with the master secret key but does not have the capabilities of public key replacement of the original signer. As in our proposed VIDS scheme, there is no facility to replace the public key of the original signer so we can treat the Type I adversary just as a dishonest user who has neither the facility to access the master secret key nor the facility to replace the public key. Hence, if both the adversaries exist in our VIDS scheme, it is obvious that Type II adversary has more attacking power than the Type I adversary. This implies that the attacking power of Type II adversary can overlap the attacking power of Type I; if Type II adversary can forge the signature of a message, it is always possible to forge the signature by the Type I adversary. From this discussion, it is sufficient to define only one adversary who is armed with the master secret key. We denote the adversary as $A$.

\subsection{Random oracles}

There are seven oracles that can be accessed by the adversary A. A brief description of these oracles is given here.

1. Create user and extract public key: Upon receiving an identity $I D \in\{0,1\}^{*}$, if the $I D$ has already been created, nothing is to be carried out. Otherwise, this oracle runs the algorithms that are components of the model to set the public key, blinding factors, binding parameters, registration $I D$ partial-private key and private key. In this case, $I D$ is said to be created. In both cases, the public key $p k_{I D}$ is returned.

2. Extract blinding factors: Upon receiving user $I D$, it returns the corresponding blinding factors if the $I D$ is already created; otherwise, it returns null.

3. Extract binding parameters: The input to this oracle is the user $I D$. If the $I D$ has already been created, it returns the corresponding binding parameters; else, it returns null.

4. Extract partial-private key: The input to this oracle is the user $I D$. If the $I D$ is already created, it returns the corresponding partial-private key; else, it returns null.

5. Extract registration $I D$ : Upon receiving user $I D$, it returns the corresponding registration identity $r e g_{I D}$ if the $I D$ is already created; otherwise, it returns null.

6. Extract private key: The input to this oracle is the user $I D$. If the $I D$ is already created, it returns the corresponding private key; else, it returns null.
7. Sign: Upon receiving user $\{I D, m\}$, it returns the corresponding signature $\sigma_{I D, m}$ in the following way:

- If the $I D$ has not been already created, it returns null.

- If the $I D$ has already been created, then it returns a valid signature $\sigma_{I D, m}$ such that

$$
\text { True } \leftarrow \operatorname{verify}\left(m, \sigma_{I D, m}, I D, p k_{I D}\right) .
$$

\subsection{The proposed game}

The game is executed between a challenger $\mathcal{C}$ and an adaptively chosen message and chosen identity adversary A.

- Set-up: The challenger $\mathcal{C}$ runs the System Parameter Generation algorithm to obtain both of the public parameter params including all public parameters. The adversary $\mathcal{A}$ is given all the public parameters along with the master secret key $s$.

- Queries: $\mathcal{A}$ Adaptively access all the oracles discussed earlier a polynomial number of times.

- Forgery: Eventually, $A$ outputs a forgery $\left(I D^{*}, P K_{I D^{*}}, m^{*}, \sigma^{*}\right)$ and wins the game if the following conditions hold true:

1. True $\leftarrow$ verify (params, $\left.m^{*}, \sigma_{I D}^{*}, I D^{*}, p k_{I D}^{*}\right)$.

2. $\left(I D^{*}, m^{*}\right)$ has never been submitted to the Sign oracle.

3. $I D^{*}$ has never been submitted to the Extract private key oracle.

4. $I D^{*}$ has never been submitted to the Extract blinding factors oracle.

\section{The proposed signature scheme}

Our proposed scheme is fitted in the model proposed by Das [16], and based on the established technique called binding-blinding technique [15]. The involved three parties in the scheme are as follows.

- PKG: The trusted authority whose role is to furnish the user with partial key after the ID of the user is verified successfully.

- Signer: Signs the message using the private key.

- Verifier: Verifies the signature using the user's public key along with the message received.

The signature generation and signature verification algorithms are based on the original identity-based signature scheme of Cha and Cheon [23]. The proposed scheme consists of the following algorithms.

- System parameter generation This randomized algorithm generates all the system parameters denoted by params, which include $G_{1}$ (an additive cyclic group of 
prime order $q$ ), $G_{2}$ (a multiplicative cyclic group of prime order $q$ ), a generator $P$ of $G_{1}$, a bilinear pairing/ map $e: G_{1} \times G_{1} \rightarrow G_{2}$, map-to-point hash function $H_{2}:\{0,1\}^{*} \rightarrow G_{1}$ and a cryptographic hash function $H_{1}:\{0,1\}^{*} \times G_{1} \rightarrow \mathbb{Z}_{q}^{*}$. In addition to these, the trusted party PKG selects $s \in \in_{R} \mathbb{Z}_{q}^{*}$ and sets its private key $s k_{P K G}=s$ and public key $p k_{P K G}=s . P$. All the public system parameters are published in the public.

\section{- User's key generation}

The following steps are carried out for calculating the private for the user using binding-blinding method, shown in figure 1 .

1. Binding parameter generation: This deterministic interactive algorithm takes both the system parameters params and signer's ID as arguments and generates binding parameters $(X, Y, Z, W)$. We note that the four binding parameters are generated using two blinding factors independently randomly chosen by the signer. After this, all the four binding parameters along with signer's $I D$ are sent to the PKG over an insecure channel.

2. Partial PKG: This deterministic interactive algorithm takes the system parameters params, binding parameters and signer's ID as arguments and outputs the partial-private key $D_{I D}$.

3. PKG: It is a deterministic interactive algorithm that takes the blinding factors and the partial-private key as input and outputs the private key $s k_{I D}$.

- Sign: This is a randomized algorithm that uses the system parameters params, the message $m$ and the private key $s k_{I D}$ as input and outputs the signature $\sigma_{(m, I D)}=(U, V)$ of the message $m$ for the identity ID. The signature generation process is shown in figure 2 .

- Verify: The signature is verified using this deterministic algorithm, which uses the params, signer's $I D$, registration token reg $_{I D}$, message $m$ and its signature $\sigma_{(m, I D)}=(U, V)$ as input, and outputs whether the signature is valid or not, as shown in figure 2. The signature is validated if the following equation holds:

$$
e(P, V)=e\left(\operatorname{reg}_{I D}, U+h \cdot p k_{I D}\right) \text {. }
$$

\subsection{Correctness}

The correctness of the proposed scheme is verified as follows:

$$
\begin{aligned}
e(P, V) & =e\left(P,(r+h) \cdot s k_{I D}\right) \\
& =e\left(P,(r+h) \cdot b \cdot s \cdot p k_{I D}\right) \\
& =e\left(b \cdot s \cdot P,(r+h) \cdot p k_{I D}\right) \\
& =e\left(r e g_{I D},(r+h) \cdot p k_{I D}\right) \\
& =e\left(r e g_{I D}, r \cdot p k_{I D}+h \cdot p k_{I D}\right) \\
& =e\left(r e g_{I D}, U+h \cdot p k_{I D}\right) .
\end{aligned}
$$

\section{Security analysis of the proposed scheme}

The security analysis of the proposed scheme is done in the ROM under adaptively chosen message attack. We have followed the same approaches used in [23, 24] for providing security proof of an identity-based digital signature scheme.

We have utilized Theorem 3 (the forking lemma) of [25] for providing security proof for our proposed scheme. According to the forking lemma, a probabilistic polynomial time algorithm $A$ can ask the maximum number of $Q_{H_{1}}$ queries to the $H_{1}$ oracle and the maximum number of $Q_{s}$ queries to the Sign oracle. Thereafter, $A$ is able to generate a

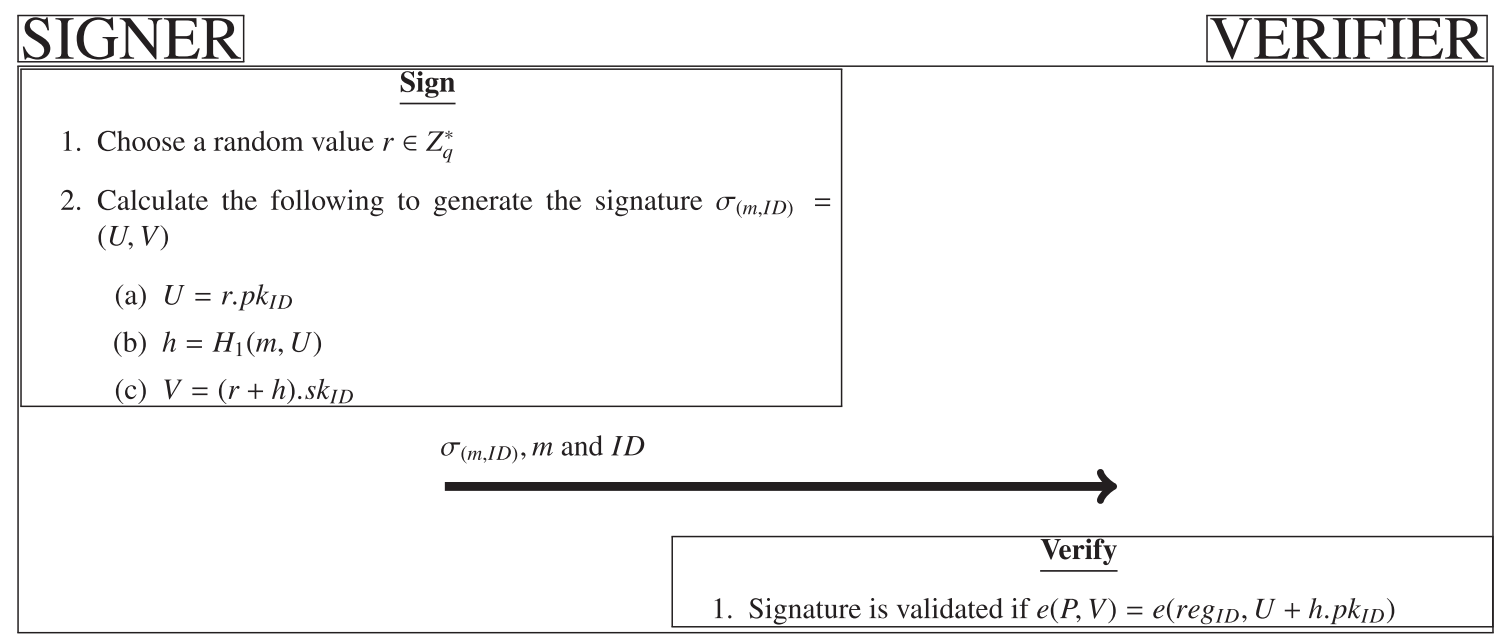

Figure 2. Signature generation and verification. 
valid signature $(m, I D, U, h, V)$ with probability $\epsilon \geq 10\left(Q_{s}+1\right)\left(Q_{s}+Q_{H_{1}}\right) / q$ and within a time-bound $t$, If the triples $(m, I D, U, h, V)$ can be simulated without knowing the private key with an indistinguishable distribution probability then there is another algorithm $B$ that can produce two valid signatures $(m, I D, U, h, V)$ and $\left(m, I D, R, h^{\prime}, V^{\prime}\right)$ such that $h \neq h^{\prime}$ in expected time $t^{\prime} \leq 120686 Q_{H_{1}} \cdot t / \epsilon$.

To apply the forking lemma, we have given a simulator Sim; in order to sign the message $m$, the simulator Sim randomly chooses $r \in Z_{q}^{*}, C \in Z_{q}^{*}, h \in Z_{q}^{*}$, then computes $U=C . P-h^{\prime} . p k_{I D}$ and $V=C . r e g_{I D}$. Now we consider the following distributions:

$$
\xi=\left[\begin{array}{c}
r \in_{R} Z_{q}^{*} \\
h \in_{R} Z_{q}^{*} \\
U=r \cdot p k_{I D} \\
V=(r+h) \cdot s k_{I D}
\end{array}\right] \quad \xi^{\prime}=\left[\begin{array}{c}
r \in_{R} Z_{q}^{*} \\
h \in_{R} Z_{q}^{*} \\
C \in_{R} Z_{q}^{*} \\
U=C \cdot P-h^{\prime} \cdot p k_{I D} \\
V=C \cdot r e g_{I D}
\end{array}\right]
$$

Let $(T, a, D)$ be a valid signature (i.e., it passes the signature verification test), where $T \in G_{1}, f \in Z_{q}^{*}, D \in G_{1}$. In the following, we show probabilities of this signature given in these distributions:

$$
\begin{aligned}
& \operatorname{Pr}_{\xi}[(U, h, V)=(T, f, D)] \\
& =\operatorname{Pr}\left[\begin{array}{c}
r \cdot p k_{I D} f=T \\
h=f \\
(r+h) \cdot s k_{I D}=D
\end{array}\right]=\frac{1}{(q-1)^{2}}, \\
& \operatorname{Pr}_{\xi}[(U, h, V=(T, f, D)] \\
& =\operatorname{Pr}\left[\begin{array}{c}
C . P-h \cdot p k_{I D}=T \\
h=f \\
C \cdot r e g_{I D}=D
\end{array}\right]=\frac{1}{(q-1)^{2}} .
\end{aligned}
$$

It can be observed that both distributions are the same, which is $\frac{1}{(q-1)^{2}}$, as the pairing group consists of $(q-1)$ number of elements and in each of the cases the $r$ and $h$ are randomly taken from the set $Z_{q}^{*}$. Hence, we can claim that without the knowledge of the private key for the signer, the signature can be simulated by simulator Sim with an indistinguishable distribution probability.

Theorem 1 Let A be a probabilistic polynomial time algorithm for adaptively chosen message attack and ID attack to our proposed scheme with advantage $\epsilon \geq 10\left(Q_{s}+\right.$ 1) $\left(Q_{H_{1}}+Q_{s}\right) \cdot Q_{H_{2}} /(q-1)$ and running time $t$, where $Q_{H_{1}}, Q_{H_{2}}$ and $Q_{s}$ are the maximum number of queries to the random oracle $H_{1}, H_{2}$ and Sign oracle asked by $A$, respectively; then $C D H P$ can be solved within expected time $t^{\prime} \leq 120686 . Q_{H_{1}} \cdot t / \epsilon$ with probability $1-1 /(q-1)$.
Proof As the forged signature is simulated with an indistinguishable distribution probability, there is an algorithm $B$ that can produce two valid signatures $(m, I D, U, h, V)$ and $\left(m, I D, U, h^{\prime}, V\right)$ such that $h=h^{\prime}$ in expected time $t^{\prime} \leq 120686 . Q_{H_{1}} . t / \epsilon$.

We can solve $C D H P$ problem from these two valid signatures $(m, I D, U, h, V)$ and $\left(m, I D, U, h^{\prime}, V^{\prime}\right)$, with probability $1-1 /(q-1)$ as follows. We run the simulator Sim using Lemma 2 in [24] with $p k_{I D}=x . P, r e g_{I D}=s . y . P$.

As signatures $(m, I D, U, h, V)$ and $\left(m, I D, U, h^{\prime}, V^{\prime}\right)$ are valid, we have

$$
\begin{aligned}
e(V, P) & =e\left(\operatorname{reg}_{I D}, U+h \cdot p k_{I D}\right) \\
& =e(\text { s.y.P, } U+h \cdot x \cdot P)=e(P, s . y \cdot U+h . s . x . y . P) \\
e\left(V^{\prime}, P\right) & =e\left(\operatorname{reg}_{I D}, U+h^{\prime} \cdot p k_{I D}\right) \\
& =e\left(\text { s.y.P }, U+h^{\prime} x . P\right)=e\left(P, s . y \cdot U+h^{\prime} . s . x . y . P\right) .
\end{aligned}
$$

Then we can have

$$
\begin{gathered}
V=s \cdot y \cdot U+h \cdot s \cdot x \cdot y \cdot P \\
V^{\prime}=\text { s.y.U }+h^{\prime} . s . x \cdot y \cdot P
\end{gathered}
$$

when $h \neq h^{\prime}$.

From Eqs. (1) and (2), we get

$$
x . y . p=s^{-1}\left(\frac{V-V^{\prime}}{h-h^{\prime}}\right) .
$$

Since both $h$ and $h^{\prime}$ are randomly chosen from $Z_{q}^{*}$, the probability of $h=h^{\prime}$ is $1 /(q-1)$. Hence, we can compute $x . y . P$ from $(P, x . P, y . P)$, i.e. solution to the $C D H P$, with probability $1-1 /(q-1)$.

\section{Efficiency analysis}

The proposed scheme is an improvement of the identity-based signature scheme of Cha and Cheon [23] utilizing bindingblinding technique to make it key escrow free as well as to eliminate the requirement of a secure channel. Our scheme is compared to an established similar type of scheme [16]. Table 1 shows the different notations of the involved operations in the schemes. Table 2 shows the efficiency comparison with [16] in terms of involved operations in each of the schemes.

\subsection{Time efficiency comparison}

We have implemented the proposed signature scheme and Das's scheme [16] on a Linux system with Intel core i3 
Table 1. Operation notation and description.

\begin{tabular}{lr}
\hline Notation & Description \\
\hline$\delta_{P O}$ & Execution of a bilinear pairing operation \\
$\delta_{P-I N V}$ & Execution of a point inversion operation in $G_{1}$ \\
$\delta_{I N V}$ & Execution of an inversion operation in $Z_{q}^{*}$ \\
$\delta_{H}$ & Execution of a general cryptographic hash function \\
$\delta_{P-A D D}$ & Execution of a point addition in the source group $G_{1}$ \\
$\delta_{E X P}$ & Execution of an exponentiation operation in the target \\
$\delta_{S M}$ & group $G_{2}$ \\
$\delta_{M U L}$ & Execution of a scalar multiplication operation in the \\
& Execution of a multiplication operation in the target \\
$\delta_{H-M T P}$ & group $G_{2}$
\end{tabular}

Table 2. Efficiency comparison.

\begin{tabular}{lcc}
\hline Schemes & Signing & Verification \\
\hline Das [16] & $\delta_{H}+2 \delta_{S M}+\delta_{P-A D D}+\delta_{E X P}$ & $2 \delta_{P O}+\delta_{H}+\delta_{P-I N V}$ \\
& & $+\delta_{E X P}+\delta_{M U L}$ \\
Proposed & $2 \delta_{S M}+\delta_{H}$ & $2 \delta_{P O}+\delta_{H}+\delta_{S M}$ \\
& & $+\delta_{P-A D D}$ \\
\hline
\end{tabular}

CPU 2.30-GHz and 2-GB RAM system configuration using Pairing-Based Cryptography (PBC) library [26] in C. We compare the proposed scheme to [16] in terms of consumed running time in the sign and verification processes.

We note that the key-generation processes in both the schemes are similar as the same binding-blinding technique is used but they are different in the signing and the verification process. Hence, it is worth giving a comparison in the processes of sign and verification. It is observed from table 3 that each of the signing and verification processes of our proposed scheme comparatively consumes lesser amount of time than the other scheme as fewer number of operations are involved in those processes. A graphical representation of the achieved results is shown in figure 3 . The size of the generated signature of these two similartype schemes is given in table 3 , where $\left|G_{1}\right|$ denotes the size of an element of the source group $G_{1}$ and lell denotes the size of an element in the set $Z_{q}^{*}$.

Table 3. Comparison of the running time.

\begin{tabular}{lccc}
\hline Scheme & Sign (ms) & Verify (ms) & Signature length \\
\hline Das [16] & 13.753 & 40.111 & $\left|G_{1}\right|+|e l|$ \\
Proposed & 6.230 & 30.641 & $2|G 1|$ \\
\hline
\end{tabular}

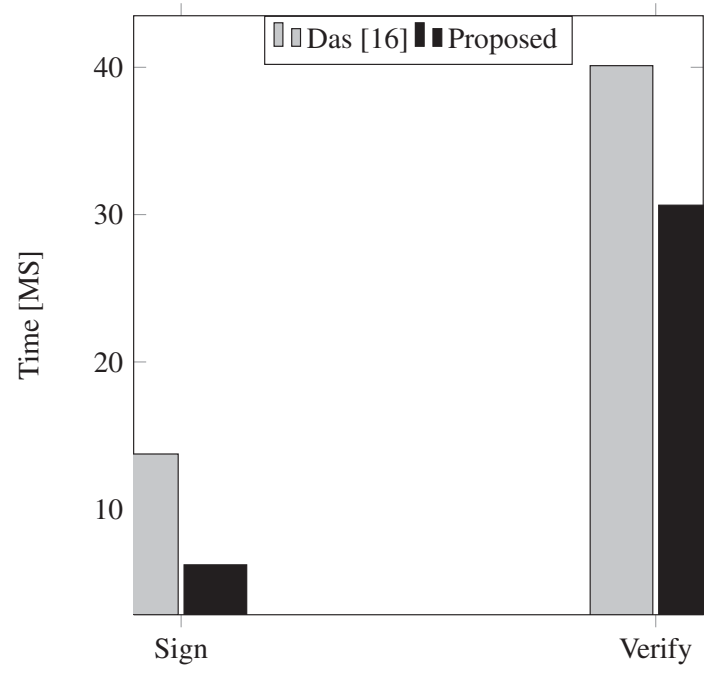

Figure 3. Comparison of the running time in sign and verify.

\section{Conclusion}

The proposed scheme is key escrow free and does not require a secure channel to transmit the private key to the user. Referring to tables 2, 3 and efficiency analysis given in section 6 , it is observed that our scheme is more computationally cost efficient than the other existing one. Moreover, the proposed scheme is secure against existential forgery under adaptively chosen message and ID attacks in the ROM assuming the intractability of the CDHP.

\section{References}

[1] Menezes A, Vanstone S and Van Oorschot P C 1996 Handbook of applied cryptography. Boca Raton, FL, USA: CRC Press

[2] Al-Riyami S and Paterson K 2003 Certificateless public key cryptography. In: Advances in Cryptology-ASIACRYPT2003, LNCS 2894, pp. 452-473

[3] Shamir A 1984 Identity-based cryptosystems and signature schemes. In: Blakeley G R and Chaum D (Eds.) Advances in Cryptology-CRYPTO, LNCS 196. Springer-Verlag, Berlin, Heidelberg, pp. 47-53

[4] Guillou L and Quisquater J J 1988 A paradoxical identitybased signature scheme resulting from zero-knowledge. In: Goldwasser $\mathrm{S}$ (Ed.) Advances in Cryptology-CRYPTO, LCNS 403. Berlin: Springer-Verlag, pp. 216-231

[5] Fiat A and Shamir A 1986 How to prove yourself: practical solutions to identification and signature problems. In: Odlyzko A M (Ed.) Advances in Cryptology-CRYPTO'86, LCNS 263. Berlin: Springer-Verlag, pp. 186-194

[6] Sahu Rajeev A and Sahadeo P 2011 ID-based signatures schemes from bilinear pairing: a survey. Front. Electr. Electron. Eng. China 6(4): 487-500

[7] Boneh D and Franklin M 2001 Identity-based encryption from the Weil pairing. In: Advances in Cryptology-CRYPTO 2001. Springer, Berlin, Heidelberg, pp. 213-229 
[8] Barreto P S L M 2007 The pairing-based crypto lounge. http://paginas.terra.com.br/informatica/paulobarreto/ pblounge.html. Accessed date 15th October, 2017

[9] Lee K and Lee D H 2014 Security analysis of an identitybased strongly unforgeable signature scheme. Inf. Sci. 286: 29-34

[10] Kar J 2014 Provably secure online/off-line identity-based signature scheme for wireless sensor network. Int. J. Netw. Secur. 16(1): 29-39

[11] Joonsang B and Yuliang Z 2004 Identity-based threshold signature scheme from the bilinear pairings. In: IEEE International Conference on Information Technology: Coding and Computing, Information Assurance and Security Track, IEEE Computer Society. ISBN: 0-7695-2108-8/04, pp. 124-128

[12] Chen L, Harrison K, Soldera D and Nigel P S 2003 Applications of multiple trust authorities in pairing based cryptosystems. In: Infrastructure Security. Berlin/Heidelberg: Springer, pp. 260-275

[13] Gentry C 2003 A certificate-based encryption and the certificate revocation problem. In: Proceedings of the International Conference on the Theory and Applications of Cryptographic Techniques. Berlin/Heidelberg: Springer, pp. 272-293

[14] Lee B, Boyd C, Dawson E, Kim K, Yang J and Yoo S 2004 Secure key issuing in ID-based cryptography. In: Proceedings of the Australian Information Security Workshop, AISW04, pp. 69-74

[15] Das M L, Saxena A and Phatak D B 2007 A proxy signature scheme with effective revocation using bilinear pairings. Arxiv preprint 07 arXiv:0712.3084
[16] Das M L 2010 A key escrow-free identity-based signature scheme without using secure channel. Cryptologia 35(1): 58-72, https://doi.org/10.1080/01611194.2010.515905

[17] Koblitz N 1994 A course in number theory and cryptography, vol. 114. Springer Science \& Business Media, UK, Berlin/Heidelberg p. 114

[18] Hankerson D, Menezes A and Vanstone S 2004 Proceedings of elliptic curve cryptography-04. Springer US

[19] Hess F 2002 An efficient identity based signature schemes based on pairings. In: Nyberg K and Heys H (Eds.) Selected Areas in Cryptography, SAC2002, LCNS 2595. Berlin: Springer-Verlag, pp. 310-324

[20] Das M L 2015 Key-escrow free multi-signature scheme using bilinear pairings. Groups Complex. Cryptol. 7(1): 47-57

[21] Pointcheval D and Jacques S 1996 Security proofs for signature schemes. In: Proceedings of Eurocrypt 1996, pp. 387-398

[22] Huang X, Yi M, Willy S, Wong D and Wei W 2007 Certificateless signature revisited. In: Information Security and Privacy. Berlin/Heidelberg: Springer, pp. 308-322

[23] Cha C J and Cheon J H 2003 An identity-based signature from gap Diffie-Hellman groups. In: Proceedings of the International Workshop on Public Key Cryptography. Berlin/ Heidelberg: Springer, pp. 18-30

[24] Huang Z, Chen K and Wang Y 2005 Efficient identity-based signatures and blind signatures. In: Proceedings of the International Conference on Cryptology and Network Security. Berlin/Heidelberg: Springer, pp. 120-133

[25] David P and Stern J 2000 Security arguments for digital signatures and blind signatures. J. Cryptol. 13(3): 361-396

[26] The pairing based cryptography (pbc) library. https://crypto. stanford.edu/pbc/. Accessed date 17th October, 2017 